\section{Successful Use of Etanercept in Acquired Angioedema in a Patient with Psoriatic Arthritis}

\section{To the Editor:}

A 57-year-old man had weekly to biweekly recurrent episodes of angioedema over 18 months, which resulted in 12 hospital admissions. He had had a seronegative arthritis compatible with psoriatic arthritis sine psoriasis for 4 years. His arthritis was active despite concomitant treatment with sulphasalzine and methotrexate. In addition, he suffered from hypercholesterolemia and hypertension, for which he was treated with rosuvastatin and lercanipidine. He suffered from ischemic heart disease and had transient cerebral ischemic attack. The episodes of angioedema were unrelated to his medications.

Laboratory investigation showed elevated creatine phosphokinase of $442 \mathrm{U} / 1$ (normal $=26-190 \mathrm{U} / \mathrm{l})$, within normal levels of lipids including cholesterol, triglycerides, and high-density lipoprotein, normal levels of complement components including $\mathrm{C} 1$ inhibitor, $\mathrm{C} 3$ and $\mathrm{C} 4$, and normal bleeding time, normal clotting time, and normal coagulation studies. With the possibility of functional abnormal $\mathrm{C} 1$ inhibitor or the presence of antibodies to the $\mathrm{C} 1$ inhibitor, he was initially treated with danazol, hexacapron, and fresh-frozen plasma during the acute episodes with no clinical benefit. The therapeutic option of bradykinin inhibitor or bradykinin receptor inhibitor was unavailable. Because his arthritis did not improve on these medications, treatment with etanercept $25 \mathrm{mg}$ subcutaneously twice a week was started. The treatment with etanercept was followed by immediate and remarkable improvement of the angioedema, with no further episodes.

Recurrent angioedema is a disorder characterized by episodes of swelling that may involve multiple anatomical locations and is caused by unregulated generation of bradykinin. Angioedema can be either hereditary (caused by deficiency in C1-INH) or acquired (caused by a functional deficiency of C1-INH), or unrelated to the complement system ${ }^{1,2}$. We report a patient with acquired angioedema treated with etanercept with complete resolution of his symptoms. The course of angioedema in this patient with normal complement components and no response to danazol, hexacapron, and FFP was typical of type III angioedema, where C1-INH is typically at normal level and function. Acquired type III angioedema may be associated with decreased synthesis of factor XII (Hageman trait), prekallikrein, and high-molecular-weight kininogen. Roles for factor XII, prekallikrein, and kininogen have been suggested in gouty arthritis.

It is conceivable that the angioedema attacks were caused by the inflammatory process secondary to the arthritis. Tumor necrosis factor-alpha (TNF- $\alpha$ ), a major inflammatory cytokine in arthritis, increases endothelial permeability, and the increased permeability of endothelial cells contributes in turn to the pathogenesis of angioedema. It was recently shown that addition of TNF- $\alpha$ and interleukin 1 beta (IL-1 $\beta$ ) neutralizing antibodies to activated peripheral blood mononuclear cells supernatant significantly reduced endothelial permeability ${ }^{3}$.

Standard treatments for hereditary angioedema, including fresh frozen plasma, danazol, and epsilon-aminocaproic acid, are usually ineffective for acquired angioedema. Likewise, newer therapies with plasma-derived C1-INH replacement or a recombinant C1-INH replacement therapy cannot abort the angioedema attacks that occur in patients with normal levels of C1-INH. More novel therapies with either a kallikrein inhibitor (ecallantide; DX-88) or a bradykinin-2-receptor antagonist (icatibant) can be suitable for all forms of angioedema, but they are not currently approved or available in most countries ${ }^{4,5}$.

Etanercept is a fusion protein composed of the p75 TNF receptor linked to the $\mathrm{Fc}$ portion of human IgG1. Etanercept exerts its action by binding to TNF in the serum, inhibiting its ability to interact with the cell surface receptors that promote the inflammatory process. The therapeutic role of etanercept in this case could be either to improve the arthritis and correct possible aberrant synthesis of kinins, or more likely by reducing the inflammation and vascular permeability.

Despite the limited data, etanercept is a promising choice as an additional therapeutic option in a subgroup of patients with type III angioedema secondary to an inflammatory disease, for which bradykinin antagonists or bradykinin receptor antagonists are unavailable. Further studies are needed to confirm this observation.

MENACHEM ROTTEM, MD, Head, Allergy, Asthma and Immunology Service; REUVEN MADER, MD, Head, Rheumatology Unit, Emek Medical Center, Afula, and Rappaport Faculty of Medicine, Technion, Haifa, Israel. Address correspondence to Dr. M. Rottem, Allergy, Asthma and Immunology Service, Emek Medical Center, Afula 18101, Israel. E-mail: menachem@rottem.net

\section{REFERENCES}

1. Széplaki G, Varga L, Szépvölgyi A, Simon K, Blaskó B, Nagy E, et al. Acquired angioedema associated with primary antiphospholipid syndrome in a patient with antithrombin III deficiency. Int Arch Allergy Immunol 2008;146:164-8.

2. Cugno M, Castelli R, Cicardi M. Angioedema due to acquired C1-inhibitor deficiency: a bridging condition between autoimmunity and lymphoproliferation. Autoimmun Rev 2008;8:156-9

3. Wakamoto S, Fujihara M, Sakagawa H, Takahashi D, Niwa K, Morioka M, et al. Endothelial permeability is increased by the supernatant of peripheral blood mononuclear cells stimulated with HLA Class II antibody. Transfusion 2008;48:2060-8.

4. Epstein TG, Bernstein JA. Current and emerging management options for hereditary angioedema in the US. Drugs 2008:68:2561-73.

5. Frank MM, Jiang H. New therapies for hereditary angioedema: disease outlook changes dramatically. J Allergy Clin Immunol 2008;121:272-80

J Rheumatol 2010;37:1; doi:10.3899/jrheum.090686 